June 28, 1962

\title{
CONTRIBUTIONS TO THE THEORY OF RANK ORDER STATISTICS: APPLICATIONS OF IATTICE THEORY
}

\author{
I. Richard Savage $1 /$ \\ Technical Report No. 15 \\ University of Minnesota \\ Minneapolis, , ...Minnesota
}

Reproduction in whole or in part is permitted for any purpose of the United States Government.

1/ Work done in part under contract Nonr-710(31), NR 042-019 of the Office of Naval Research. 
CONTRIBUTIONS TO THE THEORY OF RANK ORDER STATISTICS:

APPLICATIONS OF LATTICE THEORY

\author{
I. Richard Savage $1 /$ \\ University of Minnesota
}

0. Introduction. As a typical problem, if $x_{1}, \ldots, x_{m}$ are the observations in a first sample and $y_{1}, \ldots, y_{n}$ are the observations in a second sample, then the ranks of the $y^{\prime} s$ in the combined sample is the observed rank order. Thus the observed rank order $r$ will be a vector $\left(r_{1}, \ldots, r_{n}\right)$ consisting of an ordered subset of the integers $1, \ldots, m+n$. Corres.ponding to the observed rank order $r$ is the random variable $R$. If the $x^{\prime} s$ and $y^{\prime}$ are identically and independently distributed then for each possible $r$, one has $P(R=r)=\left(\begin{array}{c}m+n \\ n\end{array}\right)^{-1}$. On the other hand, if the $x^{\prime} s$ are a sample from a population with continuous cumblative distribution function $F($. and the $y^{\prime}$ s are a sample from a different continuous cumulative distribution function $G($.$) the rank orders will not be equally likely. In general if r \neq r^{\prime}$ there will be some choices of $F($.$) and G($.$) for which P(R=r)>P\left(R=r^{\prime}\right)$ and other choices for which $P(R=r)<P\left(R=r^{\prime}\right)$. In [3] a broad class of choices for $F($.$) and G($.$) is$ presented for which the probabilities of the rank orders, $P(R=r)$, are partially ordered. In Section 2 , this partial ordering is shown to be a distributive lattice. The associated Jordan-Dedekind distance is found to be the Wilcoxon statistic.

In Section 3, the one sample problem is considered. Again, the partial ordering (obtained in [5]) is a distributive lattice and the distance is the Wilcoxon one-sample signed-rank statistic. In Section 4, the partial ordering (obtained in [4]) for the trend problem is examined. The partial ordering is not a lattice but does satisfy the Jordan-Dedekind chain condition; the distance is Kenda11's tau (rank

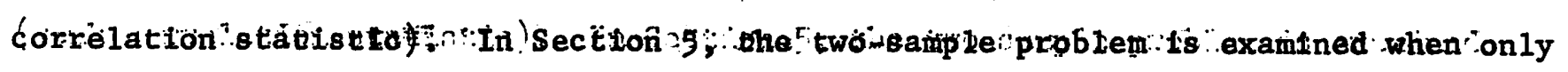

1/ Work supported in part by the Office of Nava1 Research. 
the smaller random variables are observed. In this case the partial orderings (obtaind in [2]) are of the likelihood ratios of the rank orders. The results depend on the precise form of censoring whith is being used. Section 1 contains the necessary lattice theory background for reading this paper.

This preliminary report is to formulate the kind of results which might be obtained with the aid of lattice properties. It is far from exhaustive.

1. Lattice Theory $i$ In the following $R$ is a nonempty set whose elements aradesignated by $r, s, t, r_{1}$, etc., together with twó binary operations + and $\times$ b !

Definition. $R$ is a distrdbutive lattice if it is closed under + and $x$ and:

L.1 $r \times r=r$ and $r+r=r$ for all $r$ in $R$.

L.2 $r \times s=s \times r$ and $r+s=s+r$ for all $r$ and $s$ in $R$.

L.3 $r \times(s \times t)=(r \times s) \times t$ and $r+(s+t)=(r+s)+t$ for all $r, s$, and $t$ in $R$.

L.4 $r \times(r+s)=r$ and $r+(r \times s)=r$ for all $r$ and $s$ in $R$.

L.5 (rXs) $+(r \times t)+(s \times t)=(r+s) \times(r+t) \times(s+t)$ for a11 $r, s$, and $t$ in $R$.

I. 1 through $\mathrm{L} .4$ are the lattice properties and 4.5 is the distributive property. Definition. For $r$ and $s$ in $R, r \geq s$ if $s=r x_{s}: r>s$ if $r \geqq s$ but not. $s \geqq r$.

For all r, s, and $t$ in a lattice (L.1-L.4), the following conditions are satisfled, as is easily verified:

$$
\begin{aligned}
& \text { P.1 } r \geqq r . \\
& P .2 \text { If } r \geqq s \text { and } s \geqq r \text { then } r=s . \\
& P .3 \text { If } r \geqq s \text { and } s \geqq t \text { then } r \geqq t .
\end{aligned}
$$

Also $r+s$ is the least upper bound and $r \times s$ is the greatest lower bound of the set consisting of $r$ and $s$. In a finite lattice, i.e., a lattice with a finite number of elements, there is a greatest and a least element.

a / The basic reference for this section is [1]. Diagrams illustrating the con: cepts of this section appear in the later sections of this paper.

b / For typographical convenience + and $x$ have been used instead of the conventional chp and caup. 
Definition. $r$ covers $s$ if $r>s$ and there does not exist a $t$ in $R$ such that $r>t$ $>$ s. A chain connecting $s$ to $r$ is a sequence $t_{1}, \ldots, t_{d}$ such that $s<t_{1}<\ldots<$ $t_{d}=r$, where $t_{1}$ covers and $t_{i+1}$ covers $t_{1}$ for $i=1, \ldots, d-1$. Then $d$ is said to be the length of the chain connecting $s$ to $r$. Clearly there can be several chains connecting $s$ to $r$ and they need not all have the same length. If $s \quad r$ and $R$ is finite there is plainly at least one. Jordan-Dedekind Chain Condition. For each $s$ and $r$, all chains from $s$ to $r$ are of the same length. (The length can, of course, depend on $s$ and $r_{.}$)

Throughout the remainder it is assumed that the Jordan-Dedekind conditon is met. (The condition is met in finite distributive lattices.) In a finite lattice there will be a least one chain from each element other than the smallest to the smallest element. Let $d(r)$ be the length of chains from $r$ to the smallest element if $r$ is not that element and $d(r)=0$ if $r$ is the smallest element. Let $d(R)$ be the length of the chains from the smallest to largest element. Then, for a finite distributive lattice, it is not hard to show:

A. If $r$ covers $s$ then $d(r)=d(s)+1$.

B. $d(r) \subseteq d(R)$ with equality only if $r$ is the largest element in $R$.

C. $d(R)$ is at most one less than the cardinality of $R$. If $d(R)$ is that large then the system $R$ is isomorphic to the integers $0, \ldots, d(R)$ with the interpretation $r+s=\max (r, 8)$ and $r \times s=\min (r, s)$.

D. $d(r+s)+d(s \times r)=d(r)+d(s)$.

E. $2[d(r+s+t)-d(r \times s \times t)]=d(r+s)+d(r+t)+d(s+t)-d(r \times 8)-d(r \times t)-d(s \times t)$

Definition. If $s \leqq r \leqq t$, then $r^{\prime}$ is called a relative complement of $r$ with respect to $s$ and $t$ provided $r+r^{\prime}=t$ and $r \times r^{\prime}=s$. In distributive lattices the relative complement is unique, if it exists. 
Definition. An element $r$ in $R$ is said to be join-irreducible if $r=8+t$ implies $r=s$ or $r=t$. (An element $r$ in $R$ is said to be meet-irreducible if $r=s \times t$ implies $r=s$ or $r=t$. )

In a finite distributive lattice, each element $r$ has a unique representation in the form $r=r_{1}+\ldots+r_{k}$ when $k$ (which depends on $r$ ) is made as small tas possible and the $r_{1}, \ldots, r_{k}$ are join-irreducible. The number of join-irreducible elements (excluding the smallest element) equals $d(R)$. If $r$ is join-irreducible and $r \leqq$ $r_{1}+\ldots+r_{n}$ then $r \leqq r_{i}$ for some $r_{i}$. If $r>s$ and $r$ is join-irreducible or $s$ is meet-irreducible then for $u$ such $s<u<r$ there does not exist a relative complement. In al1 of the examples (except in section 3) the largest and smallest elements each will be both join-and meet-irreducible. Hence complements will not exist--a complement being a relative complement with $\mathbf{r}$ being the largest and $\beta$ being the smallest in the lattice. In most of the examples to be considered relative complements will not exist. (Because of the location of join-irreducible elements.) 2. Two-Sample Problem. Let $\mathrm{x}_{1}, \ldots, \mathrm{x}_{\mathrm{m}}$ be independently and identically distributed with density $f(x)$ and $Y_{1}, \ldots, Y_{n}$ be distributed independently of the $X$ 's as well as being independently and identically distributed with density $g(x)$. Assume that for $x<y$

$$
\frac{f(x)}{g(x)}>\frac{f(y)}{g(y)}
$$

Let $\mathrm{W}_{1}, \ldots, \mathrm{W}_{\mathrm{m}+\mathrm{n}}$ be the $\mathrm{X}^{\prime} \mathrm{s}$ and $\mathrm{Y}^{\prime} \mathrm{s}$ when arranged from smallest to largest. Designate by $C=\left(C_{1}, \ldots, C_{n}\right)$ the ordered positions in the $W$ sequence occupied by $Y$ 's. Thus $C$ is the vector of ranks of the second sample in the combined sample. The components of C will be an ordered subset of $n$ of the first $m+n$ integers. Further let $c=\left(c_{1}, \ldots, c_{n}\right)$ be an element in the range of the random variable $c$. Write $c>c^{\prime}$ if $c_{i} \geqq c^{\prime}{ }_{i}$ for $i=1, \ldots, n$ and strict inequality holds for at least one value of $i$. Then Theorem 6.1 
of [3] is equivalent to: If $c>c^{\prime}$ then $P(C=c)>P\left(C=c^{\prime}\right)$.

To put this into the lattice framework, use the following definitions:

$$
c+c^{\prime}=\left(\max \left[c_{1}, c^{\prime}{ }_{1}\right], \ldots, \max \left[c_{n}, c_{n}^{\prime}\right]\right)
$$

and

$$
c \operatorname{krc} c^{\prime}=\left(\min \left[c_{1}, c_{1}^{\prime}\right], \ldots, \min \left[c_{n}, c_{n}^{\prime}\right]\right) \text {. }
$$

It is easily verified that $c+c^{\prime}$ and $c \times c^{\prime}$ are rank orders. The pext step is to show that these binary operations satisfy the assumptions for a distributive lattice. I.1. follows since $\max (a, a)=a$ and $\min (a, a)=a$. I.2. follows since $\max (a, b)=\max$ , $(b, a)$ and $\min (a, b)=\min (b, a)$. L.3. follows since $\max (a, \max (b, c))=\max (\max (a, b)$, c) and $\min (a, \min (b, c))=\min (\min (a, b), c)$. L.4. follows since $\min (a, \max (a, b))=a$ and $\max (a, \min (a, b))=a$. Ll5. follows $\operatorname{since} \max (\min (a, b), \min (a, c), \min (b, c))="$ the middle one of $a, b$ and $c^{\prime \prime}=\min (\max (a, b), \max (a, c), \max (b, c))$.

Next the definition of $c>c^{\prime}$ given above is equivalent to $\left(\max \left(c_{1}, c_{1}\right), \ldots\right.$, $\left.\max \left(c_{n}, c^{\prime}\right)\right)=\left(c_{1}, \ldots, c_{n}\right)$ and that $c \neq c^{\prime}$ but in terms of the lattice operations this becpmes $c+c^{\prime}=c \neq c^{\prime}$. Thus, the definition of $c>c^{\prime}$ is the lattice definition in terms of the binary operation + .

Since the number of rank orders is $\left(\begin{array}{c}m+n \\ n\end{array}\right)$, the lattice is finite. c will cover $c^{\prime}$ if their coordinates are a11 equal, except one coordinate of $c$ is one larger than the corresponding coordinate of $c^{\prime}$. The largest element is $(m+1, m+2, \ldots, m+n)$ and the smallest element is $(1,2, \ldots, n)$. If $c>c^{\prime}$ then a chain from $c^{\prime}$ to $c$ is formed by increasing a single coordinate by one unit at each step in the chain. Hence if $c>p^{\prime}$ the distance from $c^{\prime}$ to $c$ is $\sum_{l=1}^{n}\left(c_{i}-c^{\prime}{ }_{i}\right)$. So that in particular $d(c)=\sum_{i=1}^{n} c_{i}-n(n+1) / 2$ and the length of the lattice is $\sum_{i=1}^{n}(m+i)$ $-n(n+1) / 2=m$. $d(c)$ then appears as the Wilcoxon statistic (1ess a constant). The number of $c$ such that $d(c)=K$ is $\left(\begin{array}{c}m+n \\ n\end{array}\right) P(W=K+n(n+1) / 2)$ where $P($.$) is the$ distribution of the Wilcoxon statistic under the nu11 hypothesis, i.e., when the c are equally likely. 
The join-irreducible elements are of the form

$$
\left(1,2, \ldots, k-1, k, c_{k+1}, c_{k+1}+1, \ldots, c_{k+1}+n-k-1, c_{k+1}+n-k\right)
$$

where $k$ takes on the values $0,1 \ldots, n$. In more colorful language the joln-irreducible elements are those rank orders that either have one run in the second sample (the Y's) or two runs in the second sample but the smallest run is from the second sample. A join-irreducible element is characterized by the pair $\left(k, c_{k+1}\right)$. The following diagram illustrates the situation for $m=4$ and $n=3$. The higher elements are more probable. Join-irreducible, and meet-irreducible elements are designated by * and ** respectively.

$\mathrm{d}=12$

11

10

9

8

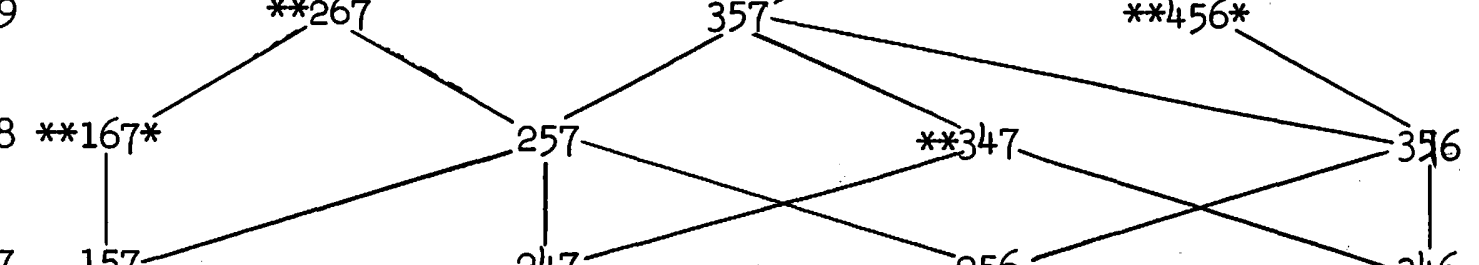

7

6

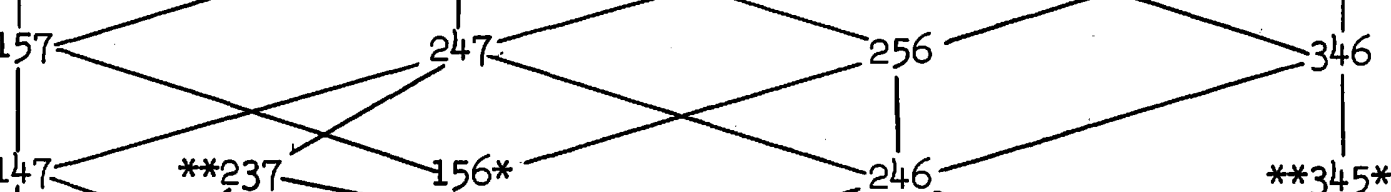

5

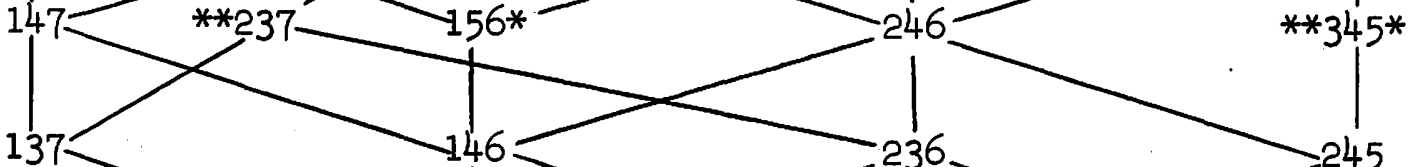

4

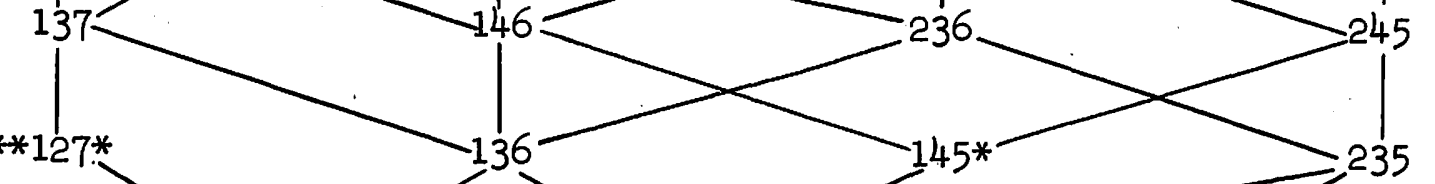

3

2

1

0

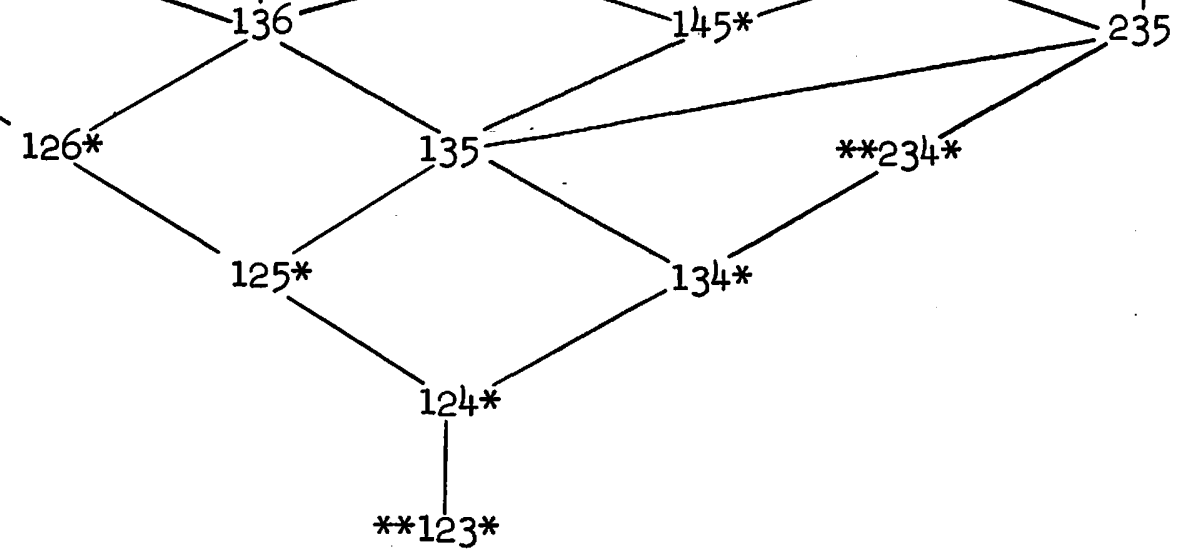


Some examples of representations of elements in terms of join-irreducible elements is given below. Notice, that at most, three are required since there are three coordinates.

$$
\begin{aligned}
& 367=167+345 \\
& 457=167+456 \\
& 267=167+234 \\
& 357=127+156+345 \\
& 257=127+156+234
\end{aligned}
$$

The number of lines in a diagram or average number of lines per element is a measure of the complexity of the diagram. A uniformly most powerful rank order test for all levels of significance will exist if the diagram is a chain. Hence it is interesting to compare the obtained partial ordering's complexity to that of a chain with the same number of elements. In general, for the two sample problem the number of lines in such a diagram is $(m+n-1)\left(\begin{array}{c}m+n-2 \\ n-1\end{array}\right)$ or the average number of lines per element is $\mathrm{m} /(\mathrm{m}+\mathrm{n})$. This is usually far from $1-\left(\begin{array}{c}\mathrm{m}+\mathrm{n} \\ \mathrm{n}\end{array}\right)^{-1}$ the value in a chain. The number of elements covered by $c$ is the number of first differences of the co-

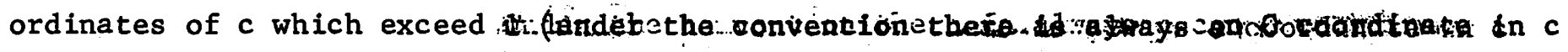
with value 0 ). The number of elements covering an element $c$ is also the number of first differences of the coordinates of $c$ which exceed 1 (under the convention there is always a $m+n+1$ coordinate with value $m+n+1)$.

Let $N(c)\left[N^{\prime}(c)\right]$ be the number of rank orders below (above) $c$ in the partial ordering. Then in forming a critical region it is desirable to put into the region those $c$ with large (sma11) values of $\mathrm{N}(\mathrm{c})\left[\mathrm{N}^{\prime}(\mathrm{c})\right]$. Unfortunately the two criteria are not compatible as will be seen by the following illustration. Let $m=2$ and $\mathrm{n}=3$, then

Case A. Criteria compatible.

$$
\begin{aligned}
& N(234)=3, N^{\prime}(234)=3 \\
& N(125)=2, N^{\prime}(125)=5
\end{aligned}
$$


Case B. Criteria incompatible.

$$
\begin{aligned}
& N(135)=4, N^{\prime}(135)=4 \\
& N(234)=3, N^{\prime}(234)=3
\end{aligned}
$$

Case C. $N^{\prime}$ criterion fails.

$$
\begin{aligned}
& N(145)=5, N^{\prime}(145)=2 \\
& N(235)=6, N^{\prime}(235)=2
\end{aligned}
$$

Case. D. N criterion fails.

$$
\begin{aligned}
& N(125)=2, N^{\prime}(125)=5 \\
& N(134)=2, N^{\prime}(134)=6
\end{aligned}
$$

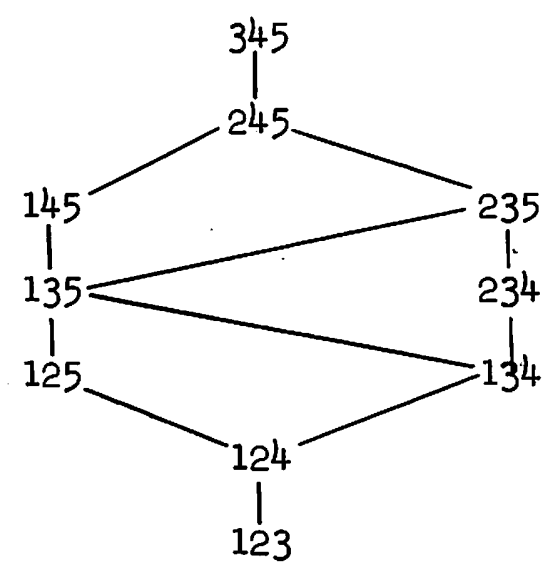

When $c>c^{\prime}$ the two criteria are compatible. The example in Case A suggests that possibly further results in describing the partial ordering could be obtained, i.e., $P(C=(234))>P(C=(125))$. In fact this conjecture is not true as can be seen from Table I, p. 608 of [3] for the case $m=n=3$ and $c=(156)$ and $c^{\prime}=(234)$.

As a special case assume $g(x)=f(x-\theta)$ where $\theta>0$ and $f(-x)=f(x)$. Then if $c$ and $c^{\prime}$ are related in the following manner (assume $m=n$ ): $c^{*}$ is the set of integers not in $c$ and $c^{\prime}$ is formed by subtracting the elements of $c^{*}$ from $m+n+1$, one has $P(C=c)=P\left(C=c^{\prime}\right)$ - see $p .975$ of [4]. Such pairs of rank orders will be said to be equivalent. 
Now to keep the lattice properties we will treat equivalent elements as a single element. In the case $m=n=3$, the diagram becomes:

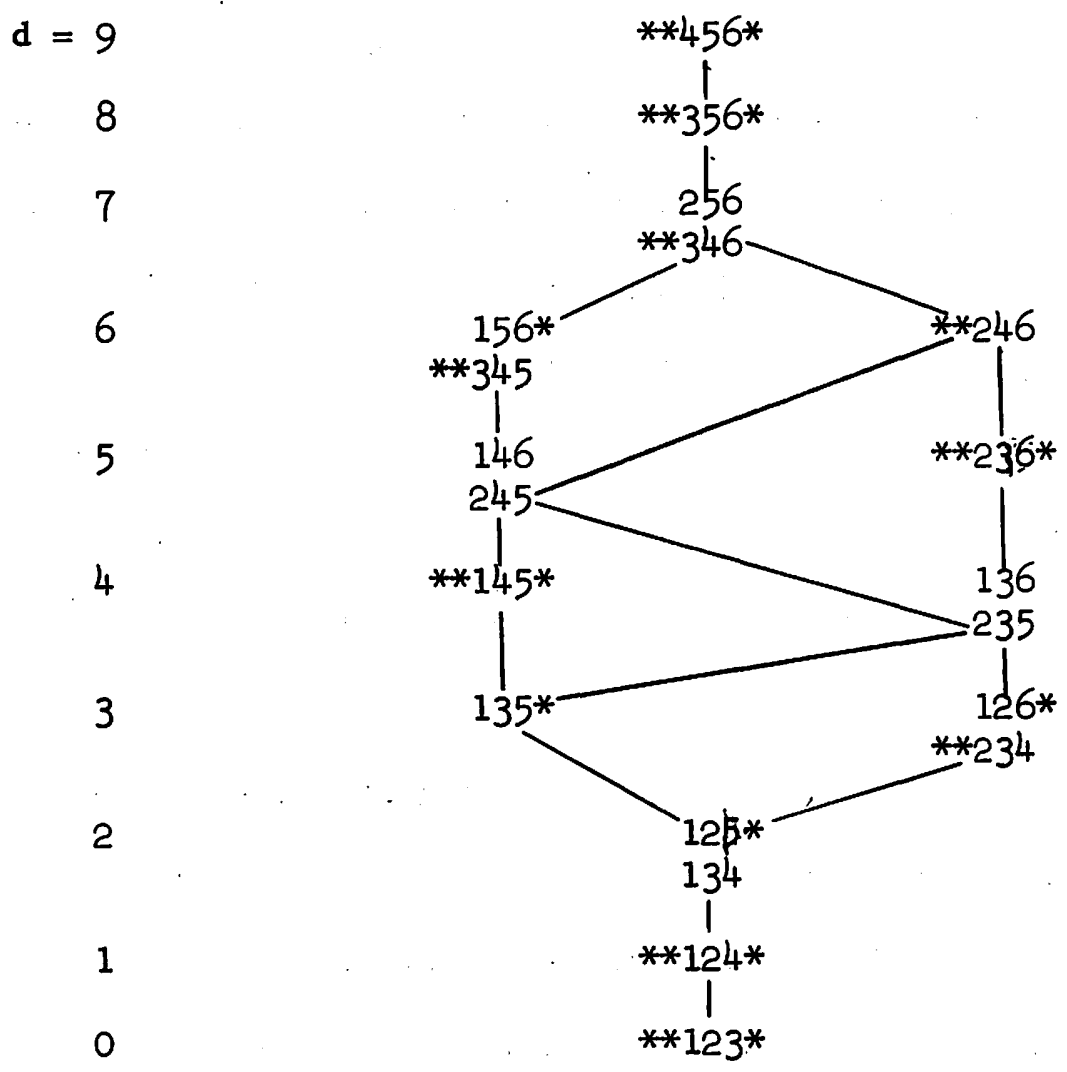

In general, the lattice will now contain $2^{n-1}+\left(\begin{array}{c}2 n \\ n\end{array}\right) / 2$ elements, $2^{n}$ elements will be of the original form, and the remainder will consist of pairs of equivalent elements. The number of lines is $\frac{(2 n-1)}{2}\left(\begin{array}{c}2 n-2 \\ n-1\end{array}\right)+2^{n-2}$.

3. One-Sample Problem. Assume $x_{1}, \ldots, x_{N}$ are independently and identically distributed with a density function $f(x, \theta)$ satisfying the following conditions:
1. $f(x, \theta)=u(x) v(\theta) e^{a(x) b(\theta)}$,
[A1though the parameter $\theta$ will not
2. $v(\theta) \geqq 0$, play a roll in the following, it is introduced to make these conditions
3. $u(x)=u(-x)>0$, appear to be the familiar ones used in talking about "exponential"
4. If $x<y$ then $a(x)<a(y)$, and families of distributions.]
5. $b(\theta)>0$.

The rank order $C=\left(C_{1}, \ldots, C_{N}\right)$ is formed in the following way: Take the absolute values of $x_{1}, \ldots, x_{N}$ and let $C_{N}$ be the rank of the largest (originally) positive 
observation in the absolute values, let $\mathrm{C}_{\mathrm{N}-1}$ be the next largest rank of the positive observations, etc. If there were originally $n$ positive observations let the first $N-n$ components of $c$ equal 0 . Then let $c=\left(c_{1}, \ldots, c_{N}\right)$ be a vector in the range of $C$. There are $2^{N}$ values of $c$ and $\left(\begin{array}{l}N \\ n\end{array}\right)$ values of $c$ with $N-n$ components equal to.0. Theorems 2.1 and 3.1 of [5] imply that if $c_{i} \geqq c^{\prime}{ }_{i}$ for $i=1, \ldots, N$ with strict inequality for some value of $i$ then $P\left(C=c^{\prime}\right)>P\left(C=c^{\prime}\right)$.

Then the lattice operations + and $x$ are exactly the same as in the two-sample case. The proof that this partial ordering; is a distributive lattice is also the same as in the two-sample case. The above definition of $>$ is consistent with these lattice operations and the definition of covering is the same as in the twosample case. The lattice is finite. The largest element is $(1,2, \ldots, N)$ and the smallest element is $(0, \ldots, 0)$. If $c>c^{\prime}$ the distance between them is $\sum_{i=1}^{N}\left(c_{i}-c^{\prime}{ }_{i}\right)$, and in particular $d(c)=\sum_{i=1}^{N} c_{i}$ and the length of the lattice $N(N+1) / 2, d(c)$ is the Wilcoxon one-sample signed rank statistic. The number of $c$ such that $d(c)=K$ is $2^{N} P(W=K)$ where $P($. ) is the null distribution of the Wilcoxon one-sampie statistic for a sample of size $\mathrm{N}$.

The join-irreducible elements are those consisting of some 0 's and a consecutive sequence of positive integers. The following diagram illustrates the situation for $\mathrm{N}=5$, where * designates join-irreducible and ** designates meetirreducible. Elements at the top are more probable. 


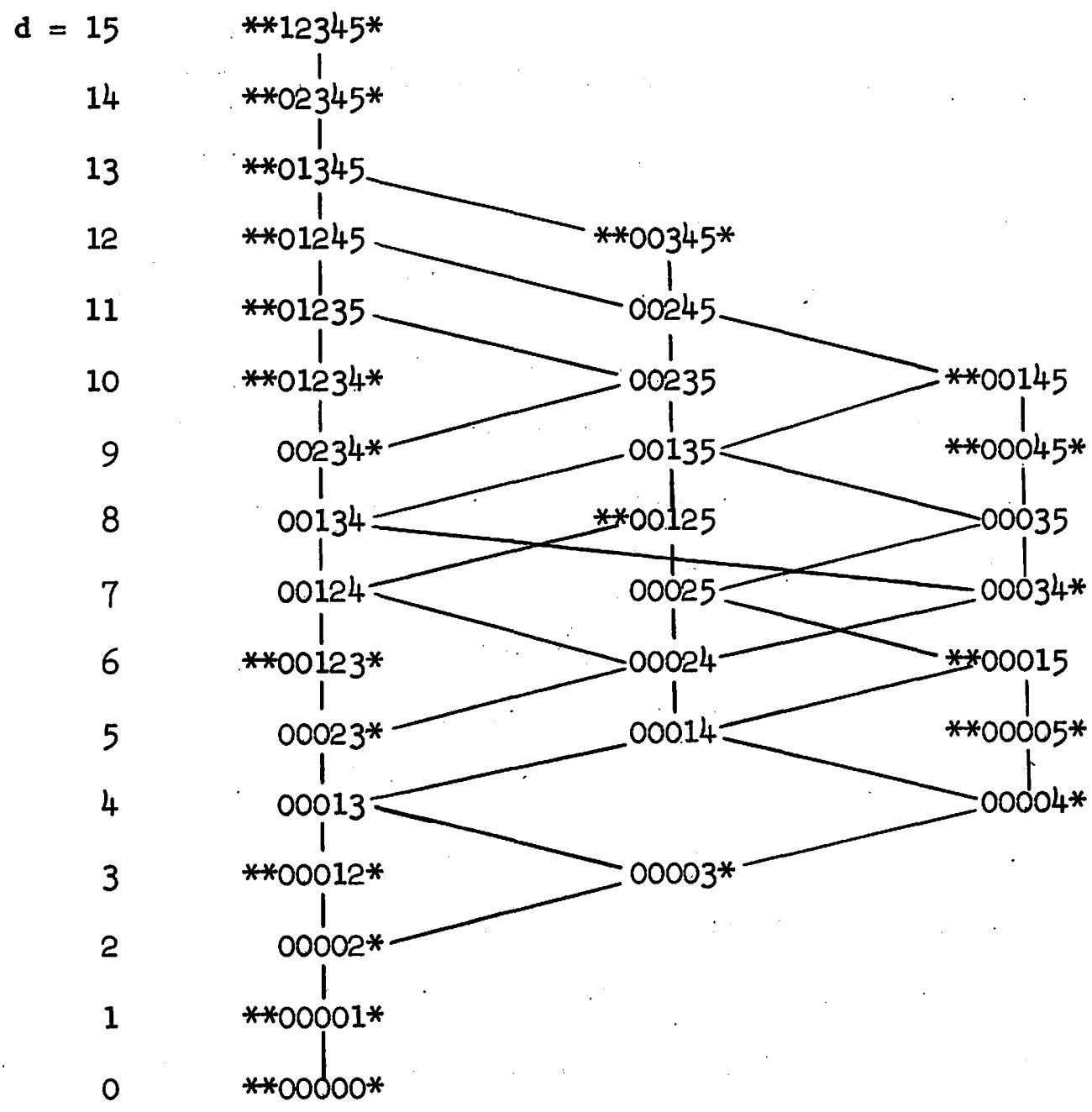

In general the number of lines in such a diagram is $(N+1) 2^{N-2}$. The average number of lines per element is $(\mathrm{N}+1) / 4$, a result comparable to the two-sample case. The number of elements covered by $c$ is one more than the number of differences between consecutive positive elements of $c$ which exceed 1 . The number of elements covering $c$ can be found by working with the dual of $c$, say $c^{*}$ where $c^{*}$ is formed from the ordered positive integers less than or equal to $\mathrm{N}$ which do not appear in c. I.e., the number of elements covering $c$ is the number of elements covered by $c^{*}$. Again, if $c^{*}$ is join-irreducible then $c$ is meet-irreducible. 
4. "Trend" Problem. Assume $\mathrm{x}_{1}, \ldots, \mathrm{x}_{\mathrm{N}}$ are independently distributed and the following conditions will be imposed on $f\left(x, \theta_{1}\right)$ the density of $x_{i}$ :

$$
\begin{aligned}
& \text { A.1. } \theta_{1} \leqq \theta_{2} \leqq \ldots \leqq \theta_{N} \cdot \\
& \text { A.2. If } \theta_{i}<\theta_{j} \text { and } x<y \text { then } f\left(x, \theta_{i}\right) f\left(y, \theta_{j}\right)-f\left(x, \theta_{j}\right) f\left(y, \theta_{i}\right) \\
& \quad \geqq 0 \text { with strict inequality for some } x<y . \\
& \text { A.3. } f(x, \theta) \text { is a continuous function in } x \text { for each } \theta_{\text {. }} \\
& \text { A.4. The set of points where } f(x, \theta)>0 \text { does not depend on } \dot{\theta} . \\
& \text { B. } f\left(x, \theta_{i}\right)=g\left(\theta_{i}\right) h(x) e^{\theta_{i} x} \text {. } \\
& \text { C. The } \theta_{i}=i \theta>0 \text { and } f\left(x, \theta_{i}\right)=f(x-i \theta)=f(i \theta-x) \text {. }
\end{aligned}
$$

The rank order $C=\left(C_{1}, \ldots, C_{N}\right)$ associated with $x_{1}, \ldots, x_{N}$ is defined in the following manner: $C_{1}$ is the index of the smallest $x_{1}, C_{2}$ is the index of second smallest $x_{1}$, $\ldots, c_{N}$ is the index of the $N$-th smallest (largest) $x_{i}$. Let $c=\left(c_{1}, \ldots, c_{N}\right)$ be an element in the range of $C$. Then there are $N$ : values of $c$, each $c$ being a permutation of the first $N$ positive integers. The rank orders $c$ and $c^{\prime}$ are said to be equivalent if $c_{i}+c^{\prime}{ }_{N-i+1}=N+1$. Under assumption $C$. equivalen $\ddagger$ rank orders are equally probable and hence when assumption $C$. is used equivalent rank orders will be treated as a single element (see Theorem 5 of [4]). Under assumption A.1 and B if two rank orders $c$ and $c^{\prime}$ are related so that

$$
\sum_{j=i}^{N}\left(c_{j}-c^{\prime}{ }_{j}\right) \geqq 0 \text { for } 1=1, \ldots, N \text { with strict inequality for some } i \text {, then }
$$
$\mathrm{P}(\mathrm{C}=\mathrm{c})>\mathrm{P}\left(\mathrm{C}=\mathrm{c}^{\prime}\right)$. (See Theorem 3 of [4]). If $\mathrm{c}$ can be obtained from $\mathrm{c}^{\prime}$ by interchanging elements of $c^{\prime}$ such that at each interchange the larger element is moved to the right (fand the smaller element to the left) then under assumption $A$. one has $P(C=c)>P\left(C=c^{\prime}\right)$. (See Corollary 1.1 of [4]). Actually all one needs to know is that the $\theta^{\prime} s$ involved in the interchanges are not all equal. 
The partial orderings associated with Theorem 3 and Corollary 1.1 do not in general generate a lattice. For instance when $\mathrm{N}=3$ the partial ordering is:

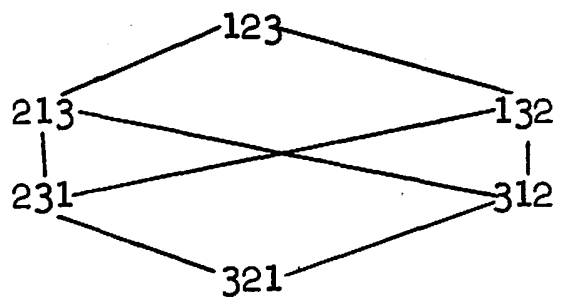

This is not a lattice since 231 and 312 do not have a unique upper bound. In the case of $\mathrm{N}=4$, situations of the same sort arise:

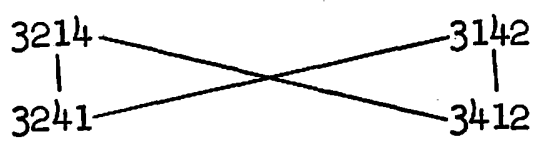

Now 3241 and 3415 do not have a unique upper bound. Nevertheless, the partial ordering from Corollary 1.1 does satisfy the Jordan-Dedekind chain condition. $d(c)$ is a linear function of Kenda11's tau, $i . e ., d(c)=\sum_{i=1}^{N}$ [number of $c_{j}$ which exceed $c_{i}$ where $j>i)$. The height of the partial ordering is $N(N-1) / 2$. Even with the use of $C$ and Theorem 5, one does not obtain a lattice -- the example for $N=4$, given above, shows this. Apparently assumption 5 and Theorem 3 only yield new results, i.e., supplemental (never contradictory) to Assumption $A$ and Corollary 1.1 when $N \geqq 5$.

The following diagram illustrates the situation for $N=4$. Again elements above dominate those below. Only the first half of the diagram is given, the other is symmetrical to it. In general the number of lines in such a diagram, e.g., just using Corollary 1.1, is given by

$$
N ![(N-1) / 2+2(N-2) / 2 \cdot 3+2(N-3) / 3 \cdot 4+\ldots+2 \cdot 1 / N(N-1)] .
$$

In particular one obtains

$\begin{array}{lc}N & \text { Number of lines } \\ 2 & 1 \\ 3 & 8 \\ 4 & 56 \\ 5 & 412\end{array}$



5. Two-Sample Problem with Censoring. "As in the two sample problem (Section 2), one has two samples of sizes $\mathrm{m}$ and $\mathrm{n}$ respectively. Now, however, a rule is specified so that only the smaller observations are obtained. If in fact $\mathrm{N}^{*}$ observations are obtained and of these $\mathrm{m}^{*}$ are from the first and $\mathrm{n}^{*}$ are from the second sample, a rankforder is formed as in the two sample problem but m* will be added as a new first component. Thus if one observed from the first sample $(-3,8,4)$ and from the second sample $(2,9)$ the rank order would be $c=(3 ; 2,5)$. The following restrictions are obvious $\mathrm{m}^{*} \leqq m, \mathrm{n}^{*} \leqq \mathrm{n}, \mathrm{m}^{*}+\mathrm{n}^{*}=\mathrm{N}^{*} \leqq m+n$, the coordinates of $c$ following the ";" are a subset of $n^{*}$ of the smaller $m^{*}+n^{*}$ positive integers, and if $c$ has only one component there were no observations obtained from second sample. Certain rank orders are redundant, $i . e .$, once $n^{*}=n$ or $m^{*}=m$ one knows which additional rank orders will be obtained if further observations are collected. Section 2 of [2] describes in detain the numbers of rank orders and numbers of non-redundant rank orders for various consoring schemes. In this case with the assumption made in section 2 , the rank orders are not equi-probable. However, if each rank order probability is divided by its probability under the null hypothesis to obtain the likelihood ratio of the rank orders then the following partial ordering of the likelihood ratios is obtained. (Rules b. and c. shquild not be applied to pairs of redundant rank orders:):

a. If $c$ and $c^{\prime}$ are such that $m^{*}=m^{\prime *}$ and $c_{i} \geqq c^{\prime}{ }_{i}$ for $i=1, \ldots$, $\mathrm{n} *\left(=\mathrm{n}^{\prime *}\right)$ then write $c>\mathrm{c}^{\prime}$ and the likelihood ratio of $\mathrm{c}$ is greater than that of $c^{\prime}$.

b. If $c$ and $c^{\prime}$ are identical except $m^{*}>m^{*}$ then write $c>c^{\prime}$ and the likelihood ratio of $c$ is greater than that of $c^{\prime}$.

c. If for $c$ and $c^{\prime}$ one has $m^{*}=m^{*}, n^{*}<n^{* \prime}$, and $c_{i}=c^{\prime}$ for $i=1,2, \ldots, \min \left(n *, n^{\prime}\right)$ then write $c>c^{\prime}$ and the 1ikeli-. hood ratio of $c$ is larger than that of $c^{\prime}$.

Clearly this is a more complicated partial ordering than those considered previous1y. 
An application of $\mathrm{b}$. and then $\mathrm{c}$. yields $(2 ; 2)>(1 ; 2,3)$, i.e., $(2 ; 2)>(1 ; 2)$ $>(1 ; 2,3)$. Hence there is more to the partial ordering for fixed $N^{*}$ than a. would indicate.

In discussing the partial orders, one can either work with all of the rank orders or those that waüld appear under a specific censoring scheme.

In the general case it is easy to show that not all chains between a given pair of points have the same length. Consider chains between $(3)$ and $(1 ; 2)$. The intervening links for one chain are (2), (1), and for another chain (2), (2;3), $(2 ; 2)$. This implies the entire structure does not have the distributive property.

Considering all of the rank orders as a whole the structure does form a lattice in the following manner:

\section{Define:}

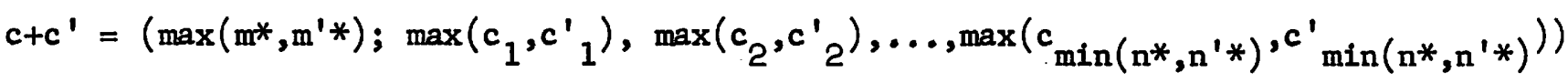
cxc' is defined in a dual manner, $1 . e .$, replace $m i n$ by $\max$ and $\max$ by min throughout. These definitions will yield the same ordering as given in a., b., and c. Also one can verify that the assumptions for a lattice are satisfied.

As a special case assume $N^{*}$ is fixed and that $N^{*}<\min (m, n)$. Now it can be shown that the lattice is distributive. The distance to the origin from $c=\left(\mathrm{m}^{*} ; c_{1}\right.$, $\ldots, c_{N *-m^{*}}$ ) is given by

$$
\left(\mathrm{N}^{*}+1\right)\left[\mathrm{m}^{*}-\frac{\mathrm{N}^{*}}{2}\right]+\sum_{i=1}^{\mathrm{N}^{*}-\mathrm{m}^{*}} \mathrm{c}_{1}
$$

This is closely related to the statistic of corollary 3.5 of [2], and essentially is the Wilcoxon statistic with a "correction for censoring". In structures of this form there are $2^{N^{*}}$ rank orders, and the length of the structure is $N^{*}\left(N^{*}+1\right) / 2$. It is easily verified that the structure is a sublattice of the lattice of all rank orders, i.e., it is a lattice with the same + and $x$ operations as the lattice for all of the rank orders. The number of lines in these diagrams are

$$
2^{N^{*}-2}\left(N^{*}+1\right) \text {. }
$$


The following illustrates the partial ordering for $N^{*}=5$ and $5<\min (m, n)$ (large likelihood ratios are at the top):

$\mathrm{d}=15$

14

13

12

11

10

9

8

7

6

5

4

3

2

1

0
(5)
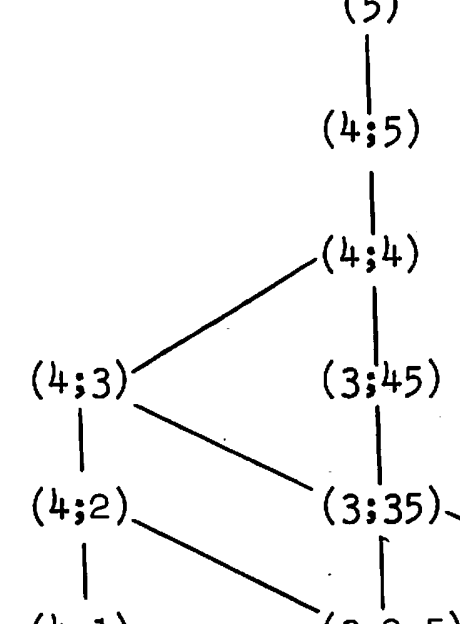

$(4 ; 1)$

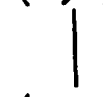

$(3 ; 15)^{\prime}$

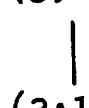

$(3 ; 1$

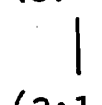

$(3 ; 13)>(2 ; 235)$

1

$(3 ; 12)$

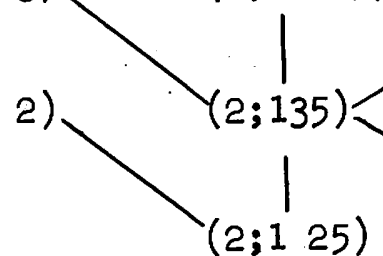

1

$(2 ; 124)$

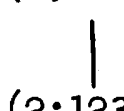

(2;123)

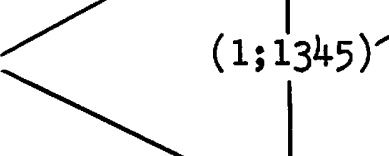

(2;345)
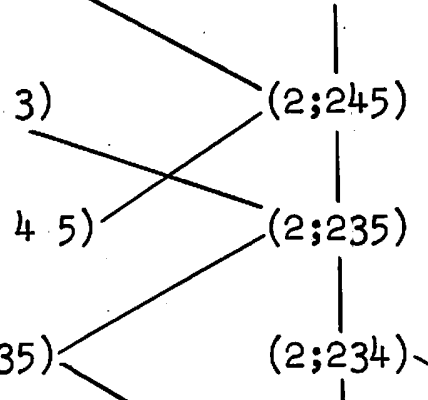

$>_{(2 ; 134)}$
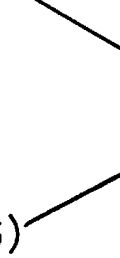

$>_{(1 ; 2345)}$

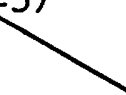

(1;1235)

(1;1234)

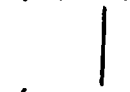

$(0 ; 12345)$ 
As another censoring scheme, assume $n^{*}=k$ where $k \leqq n$, and thus $m^{*}$ and $N^{*}$ are random variables, but $\mathrm{n} *$ is fixed. To illustrate the situation consider theflcase where $m=4$, and $k=2$. Then:

$\mathrm{d}=8$

7
6
5
4
3
2
1
0

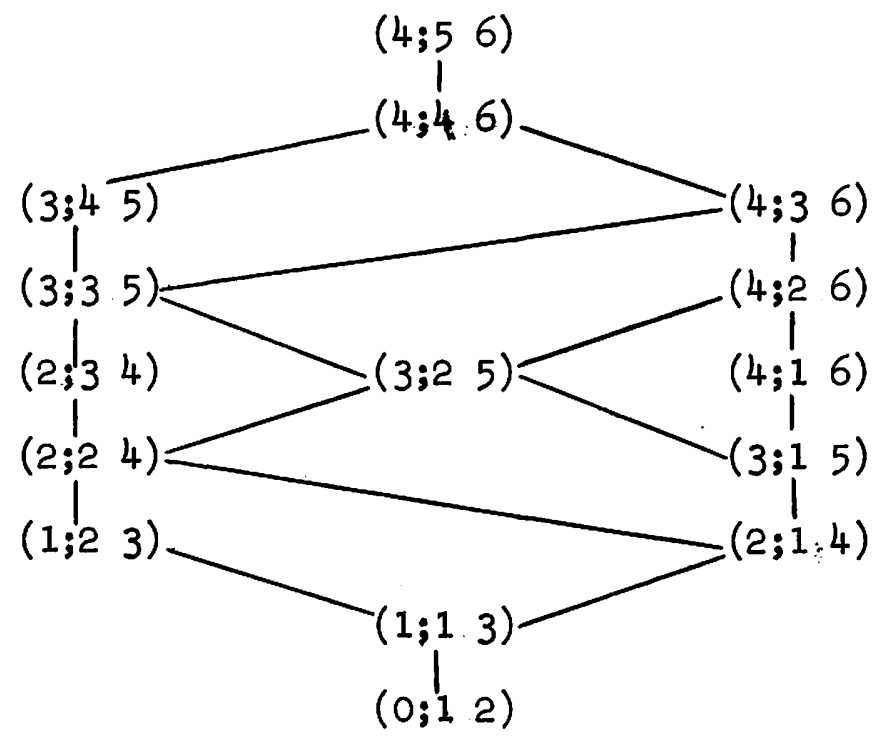

In this case the structure is isomorphic to the structure for the two-sample problem, where the first sample size is $m$ and the second sample size is $k$. However, here we are talking about likelihood ratios in contrast to probabilities (the situation for the two-sample problem). 


\section{APPENDIX: Some Questions}

1. If $\mathrm{d}(\mathrm{c})=\mathrm{d}\left(\mathrm{c}^{\prime}\right)$ can one bound $\left|\mathrm{N}(\mathrm{c})-\mathrm{N}\left(\mathrm{c}^{\prime}\right)\right|$ ?

2. Are there further details of the partial orderings which have been described here which could be deduced from the present assumptions or slight modifications of them? We know for instance that in the two-sample problem for case of exponential alternatives that the partial ordering has more detail, and the same is true for normal alternatives in the one-sample problem.

3. What statistical interpretation could be attached to such concepts as joinirreducible?

4. Are there more interesting censoring schemes than those discussed in Section 5 ?

5. There are other natural partial orderings to consider, e.g., the two-sample problem with two-sided alternatives. Will these yield lattices?

6. What kinds of assumptions would be required for the "trend" problem in order to obtain a lattice?

7. Are there other lattice concepts, particularly quantitative ones which would be of interest here? 


\section{REFERENCES}

[1] Birkhoff, Garrett (1961), Lattice Theory (Revised Edition), American Mathematical Society, Providence, xili, 283.

[2] Rao, U. V. R.; Savage, I. R.; Sobe1, M. (1960), Contributions to the theory of rank order statistics: the two-sample censored case, Ann. Math. Statist., 31, 415-426.

[3] Savage, I. Richard (1956), Contributions to the theory of rank order statistics: the two-sample case, Ann. Math. Statist., 27, 590-615.

[4] Savage, I. Richard (1957), Contributions to the theory of rank order statistics: the "trend" case, Ann. Math. Statist., 28, 968-977.

[5] Savage, I. Richard (1959), Contributions to the theory of rank - order statistics: the one-sample case, Ann. Math. Statist., 30, 1018-1023. . 